\title{
Tratamiento biorregulador de la Tenosinovitis del Tibial Posterior. A propósito de un caso
}

\author{
Bio-treatment of Tibials Posteriori Tenosynovitis. A Case Report
}

\author{
Fernando Ares Bella
}

Diplomado en Podología

ortoares@hotmail.com

Correspondencia:

D. Fernando Ares Bella

Clínica de Podología Roma

Plaza Manuel Becerra, 18

E-28028 Madrid

Fecha de recepción: 10 de febrero de 2011

Fecha de aceptación: 22 de junio de 2011

El autor declara no tener ningún tipo de interés económico o comercial.

\section{RESUMEN}

Objetivos: Valorar la eficacia de Medicamentos Biorreguladores y la Biopuntura, en el tratamiento de la Tenosinovitis del Tibial Posterior, y su adecuación a la consulta de Podología Deportiva.

Material y Métodos: Hacemos el seguimiento de un caso clínico a lo largo de un año verificando el grado de recuperación funcional obtenido. El protocolo terapéutico consta de sesiones de Biopuntura, infiltración terapéutica con Biomedicamentos, en el trayecto del tendón del Tibial Posterior y soporte oral.

Resultados: partiendo de la inactividad del corredor, tras un año de tratamiento, el paciente recupera su actividad deportiva y valora, utilizando Escalas Analógicas Visuales (VAS), la Recuperación Funcional obtenida con una puntuación (1-10) de 8. No se ha registrado ningún efecto secundario, ni errores en la inserción del protocolo. El grado de eficiencia y eficacia, los niveles de seguridad en el empleo y manejo de los Biomedicamentos son excelentes.

Conclusiones: la Biopuntura aporta una perspectiva holística de tratamiento en patologías tan complejas como la Tenosinovitis del Tibial Posterior, y se muestra como un método válido para su tratamiento, de fácil manejo técnico para el Podólogo y un elevado grado de seguridad al carecer, prácticamente de efectos secundarios.

Palabras clave: inflamación; tenosinovitis; tibial posterior; entesitis; biorregulación; biopuntura; pie.

\section{ABSTRACT}

Objectives: To evaluate the efficacy Bioregulators Drugs and Biopunture in the treatment of the posterior tibial tenosynovitis, and its relevance to the query of Podiatric Sports.

Material and Methods: We track a case report over a year checking the degree of functional recovery achieved. The therapeutic protocol consists of Biopuntura sessions, therapeutic biomedicines infiltration in the course of the tendon of tibialis posterior and oral support.

Results: starting from the inactivity of the runner, after a year of treatment, the patient returns to its sporting activities and values, using Visual Analogue Scales (VAS), functional recovery obtained with a score (1-10), 8. There has been no side effects, or errors in the insertion of the protocol. The degree of efficiency and effectiveness, levels of job security and management of bio-medicines are excellent.

Conclusions: Biopuntura provides a holistic view of treatment in diseases as complex as tenosynovitis of the tibialis posterior, and is shown as a valid method for processing, user-friendly technical support for the Podiatrist and a high level of security to lack, almost side effects.

Key words: inflammation; tenosynovitis; tibialis posteriori; enthesithys; bioregulation; biopunture; foot.

Sumario: 1. Introducción. 2. Recuerdo anatómico. 3. Fisiopatología. 4. Bases para el tratamiento biorregulador. 5. Caso clínico. 6. Conclusiones. Bibliografía

Referencia normalizada: Ares Bella, F. Tratamiento biorregulador de la Tenosinovitis del Tibial Posterior. A propósito de un caso. Rev. Int. Cienc. Podol. 2013; 7(2):77-82. 


\section{INTRODUCCIÓN}

El tendón del Tibial Posterior es una estructura fundamental para la estabilidad del tobillo y del arco longitudinal, que participa de manera activa en la flexión plantar del tobillo con adducción, supinación y valoración del arco plantar y actúa junto al resto de músculos retromaleolares, como ligamento interno activo del tobillo. Su lesión, aunque menos frecuente que la de los tendones peroneos o del Tendón de Aquiles, genera un compromiso importante frente al desarrollo de la actividad deportiva, y su tratamiento precoz asegura al deportista la total recuperación del tendón y de su funcionalidad.

\section{RECUERDO ANATÓMICO}

El Músculo Tibial Posterior es un músculo semipenniforme, es el más interno de los músculos retromaleolares y discurre desde su origen en la cara posterior de la tibia, en la parte externa y en la cara posterior de la aponeurosis peronea-tibial, hacia abajo y hacia dentro, pasa por detrás del maléolo tibial por los canales cubiertos por el retinaculum posterior de los flexores. Finaliza en el tubérculo del escafoides, los cuneiformes, el cuboides y los metatarsianos medios. Su importancia mecánica radica en la sinergia con la que interactúa con el Triceps Sural, aportando estabilidad al tobillo durante el esfuerzo de base de extensión. Está inervado por el Tibial Posterior L5-S1-S2. Participa en la flexión plantar del tobillo con aducción, supinación y valoración del arco plantar.

\section{FISIOPATOLOGÍA}

La función principal de la vaina sinovial es asegurar el deslizamiento del tendón durante el movimiento, asegurando así la estabilidad. El sobreuso del tendón puede generar la fricción de la vaina contra la estructura ósea que intenta salvar ${ }^{1}$, su inflamación y la consiguiente formación de subproductos de características pegajosas que tienden a hacer que el tendón deslizante se adhiera a la membrana sinovial ${ }^{2}$, detectándose además exceso de producción de colágeno, en un intento de reparación tisular que asegure las características del tendón de extensibilidad y flexibilidad, pero que resulta en una fibrosis y la consabida rigidez ${ }^{3,4}$. La situación que puede llevar a desencadenar este cuadro responde a múltiples factores que de manera genérica podemos resumir en causas traumáticas (golpe directo/indirecto, estado de la pista -parece que el exceso de partes inclinadas en la pista influiría en la presencia de esta patología-, calzado inadecuado...) causas biomecánicas por defecto de forma del tendón del TP, en los que deformaciones de retropié, especialmente el pie pronado, marcaría la predisposición a la insuficiencia de este tendón, como una combinación de ambas o bien como resultado de la presencia de una enfermedad sistémica.

El resultado es una clínica dolorosa que impide la actividad deportiva . Podemos encontrar por añadidura y con frecuencia, edema perimaleolar como resultado del secuestro inflamatorio, y periostitis en las áreas de Unión Osteotendinosa ${ }^{5}$, definiendo en conjunto una entesitis de inserción que compromete todas las estructuras del "órgano articular", es decir ligamentos, cápsula articular, hueso (porción mineralizada) y, por supuesto, el tendón.

\section{BASES PARA EL TRATAMIENTO BIORREGULADOR}

Dada la complejidad de tratamiento de un segmento sometido a un trabajo como el que realiza el TP, las mejores opciones pasan por un tratamiento precoz, una vez producida la lesión.

El protocolo de actuación en Medicina Deportiva marca como medida inicial de choque, ante el traumatismo durante la actividad deportiva, cuatro acciones conocidas por sus siglas en inglés RICE (reposo, hielo, elevación y compresión). Una vez superada esta etapa inicial, esencial para el desarrollo posterior del proceso inflamatorio, valoraremos el daño sufrido por el Tendón del Tibial Posterior e implantaremos las medidas terapéuticas.

Es imprescindible realizar un diagnóstico diferencial en el que se valora la rotura parcial/ total del tendón, su posible desinserción o bien si la lesión se ha producido como consecuencia 
del sobreuso/sobrecarga del tendón. En todos los casos, las medidas terapéuticas van encaminadas a corregir factores mantenedores (alteraciones biomecánicas, corregir carencias nutricionales, alteraciones hormonales y del sueño) y, como elemento común a cualquier lesión, a manejar la evolución del proceso inflamatorio.

\section{El medicamento Biorregulador}

Por definición el MB son productos médicos que no suprimen las reacciones neuroinmunológicas ni interfieren con los sistemas fisiológicos de defensa del organismo. Sus objetivos terapéuticos son estimular la curación natural, regular los procesos inflamatorios, regenerar el tejido dañado, estimular la circulación sanguínea, regular los reflejos neurales y estimular los mecanismos de detoxificación. Tienen dos características estructurales fundamentales, organotropismo (afinidad por el tejido sobre el que actúan) y tropismo funcional (especificidad sobre el proceso sobre el que se actúa) ${ }^{6}$.

El medicamento que figura en la Tabla 1, es el principal agente bioterapéutico en Medicina Deportiva para el tratamiento de lesiones. Su principal virtud es la estimulación de las TFG- $\beta$, principal modulador de la vía Tregulador. Son de uso corriente dos medicamentos más, siguiendo los pilares de la Medicina Biorreguladora, junto con la Inmunomodulación, un medicamento para el drenaje y detoxificación (Tabla 2), y el soporte celular especifico del tejido lesionado (Tabla 3). Esto aporta otro concepto como es el de coctel terapéutico, que amplifica las posibilidades terapéuticas y disminuye ostensiblemente los tiempos de recuperación.

Tabla 1.MB para el tratamiento de la inflamación aguda.

\section{Solución inyectable: $\mathbf{2 , 2} \mathbf{m l}$ contienen:}

Arnica D 2, Calendula D 2, Chamomilla D 3, Symphytum D 6, Millefolium D 3, Belladonna D 2 ana 2,2 $\mu \mathrm{l}$; Aconitum D 2 1,32 $\mu \mathrm{l}$; Bellis perennis D $21,1 \mu \mathrm{l}$; Hypericum D 2 0,66 $\mu$; Echinacea angustifolia D 2, Echinacea purpurea D 2 ana $0,55 \mu \mathrm{l}$; Hamamelis D $10,22 \mu \mathrm{l}$; Mercurius solubilis Hahnemanni D $61,1 \mu \mathrm{g}$; Hepar sulfuris D $62,2 \mu 1$.

Tabla 2 MB para la Detoxificación y el Drenaje

Solución inyectable: $2,2 \mathrm{ml}$ contienen:

Arnica D 2, Calendula D 2 ,

Chamomilla D 3, Symphytum D 6, Millefolium D 3, Belladonna D 2 ana 2,2 $\mu \mathrm{l}$; Aconitum D 2 1,32 $\mu \mathrm{l}$; Bellis perennis D $21,1 \mu \mathrm{l}$; Hypericum D $20,66 \mu$; Echinacea angustifolia D 2, Echinacea purpurea D 2 ana $0,55 \mu \mathrm{l}$; Hamamelis D $10,22 \mu \mathrm{l}$; Mercurius solubilis Hahnemanni D $61,1 \mu \mathrm{g}$; Hepar sulfuris D $62,2 \mu 1$.

Tabla 3. MB para lesiones musculares y miotendinosas

Solución inyectable: $1,1 \mathrm{ml}$ contiene:

Myosotis arvensis D 3, Veronica

D 3, Teucrium scorodonia D 3, Pinus sylvestris D 4, Gentiana lutea

D 5, Equisetum hyemale D 4, Sarsaparilla D 6, Scrophularia nodosa

D 3, Juglans regia D 3, Calcium phosphoricum D 12, Natrium sulfuricum

D 4, Fumaria officinalis D 4, Levothyroxinum D 12, Aranea diadema

D 6 ana $0,55 \mu \mathrm{l}$; Geranium robertianum D 4, Nasturtium aquaticum

D 4, Ferrum jodatum D 12 ana $1,1 \mu \mathrm{l}$. 


\section{La Biopuntura}

A menudo confundida con la Mesoterapia, su principal diferencia es el uso exclusivo de agentes bioterapéuticos. Además, la biopuntura utiliza más técnicas de infiltración que la Mesoterapia, de mayor profundidad, como la infiltración intraarticular o la intralesional. $\mathrm{Su}$ uso, a diferencia de otras vías de administración, nos aporta, en primer lugar la toma de contacto del agente bioterapéutico con el tejido lesionado lo que significa un inicio precoz de la acción terapéutica al no tener que pasar por tracto gastrointestinal. La infiltración local nos aporta tres perspectivas diferentes en cuanto a reactividad: segmental, neurológica y neuromuscular. Además, por técnicas como la Acupuntura, sabemos que la punción local implica una serie de reacciones a nivel del sistema inmunológico-cualquier agresión tisular implica el inicio de la cascada inflamatoria con el fin de reparar el tejido y por tanto la reactivación de los procesos fisiológicos de curación- y el desencadenamiento de reacciones neurológicas como son la liberación de endorfinas-que provocan la inhibición presináptica de la primera neurona impidiendo la transmisión de la información/dolor, o bien post sináptica, que libera encefalinas y dinorfinas, y bloquea los diferentes neurotransmisores-.

La técnica más común para el tratamiento de lesiones osteomusculares en el pie son las infiltraciones subcutáneas y las intradérmicas, las cuales responden a los principios antes citados.

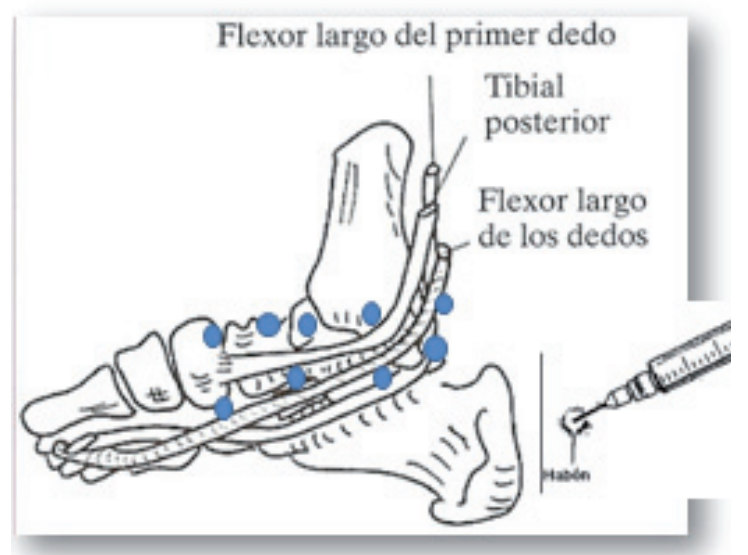

Ilustración 1. Puntos de inserción de Medicamento Biorregulador en el trayecto del tendón del TP.

\section{Protocolo de actuación}

En la Tenosinovitis del TP se depositan pápulas de bioterapéutico en diferentes localizaciones a lo largo del trayecto del tendón de inserción del Tibial Posterior (Ilustración 1). La técnica que empleamos es la infiltración intradérmica, dado la superficialidad del musculo tibial posterior y con el fin de eliminar, en la medida de lo posible, molestias al paciente. Utilizamos jeringas de $5 \mathrm{ml}$ en donde mezclamos el contenido de los tres agentes bioterapéuticos citados anteriormente, con agujas de 30G. La técnica se ejecuta siguiendo los canones de actuación de una infiltración convencional. Verificamos la situación del segmento lesionado mediante clínica, debe desaparecer el dolor y la impotencia funcional, el paciente aporta su propia valoración a través de VAS (Visual Analogic Scale) y hacemos el seguimiento de rendimiento durante la actividad deportiva.

\section{CASO CLÍNICO}

El paciente es un hombre, de 34 años de edad, 1,72 de estatura y 80 kilos. Constitución atlética, desarrolla su profesión como docente en un Instituto de la Comunidad Valenciana. Durante toda su juventud ha practicado el atletismo, desarrollando mayor trabajo en gimnasio, trabajo excéntrico, en los últimos años. Ha sufrido diversas lesiones de diferente consideración en miembro superior e inferior, siendo éstas atendidas con distinta evolución. En Octubre de 2008 acude a mi consulta a través de un compañero; el paciente presenta un cuadro doloroso agudo en zona interna de retropié que se extiende hacia proximal, que le impide desarrollar cualquier tipo de actividad deportiva. Ha pasado por consulta de Trauma, por Fisioterapia (diatermia y onda corta), se ha negado a infiltrarse corticoides e incluso le sugieren la posibilidad de cirugía.

Se le practica una primera exploración clínica, en el que detectamos el dolor a la palpación en el trayecto del TP, edema periestructural y retromaleolar e impotencia funcional. El paciente aporta RMN (Ilustración 2), en la que podemos apreciar la alteración de la vaina del tendón y la acumulación de líquido en torno al maléolo tibial. 
Se instaura el protocolo de actuación con Biopuntura, 1 sesión por semana, durante 4 semanas (Ilustración 3). Durante un año el paciente se somete a infiltración una vez al mes. Detallamos la evolución del paciente en la Tabla 4.

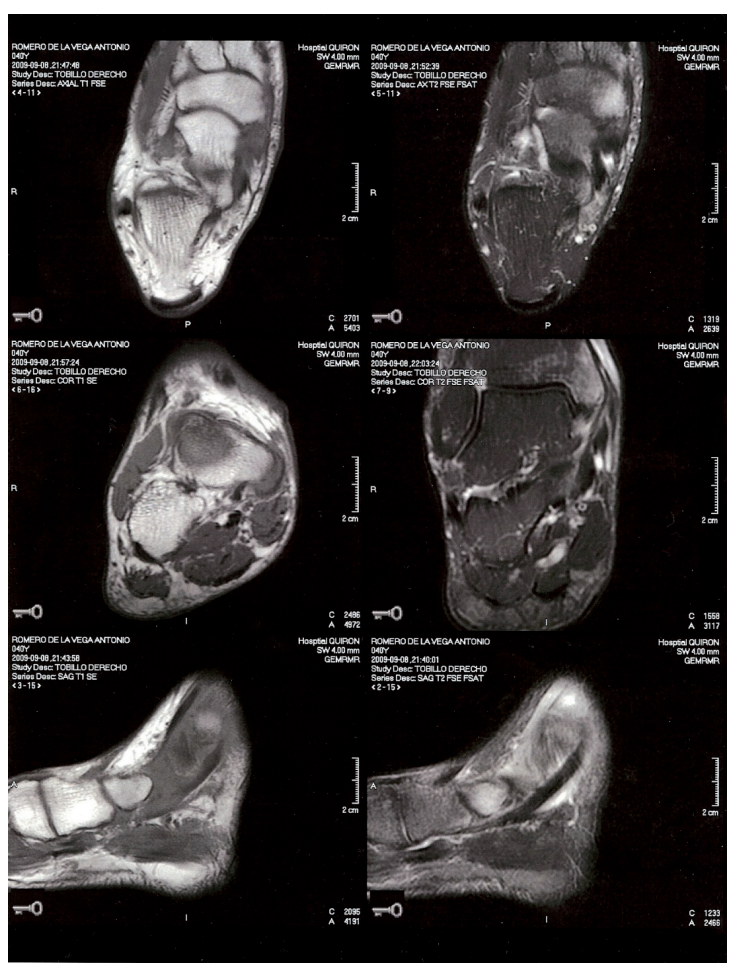

Ilustración 2. RMN donde se aprecia la alteración del tendón del TP y el acúmulo de líquido en torno al maléolo tibial.
A medida que avanza el tratamiento se observa la mejora de la condición física y del rendimiento deportivo. A partir de la cuarta semana de tratamiento se añaden medidas correctoras de la desviación biomecánica y trabajo de fisio-

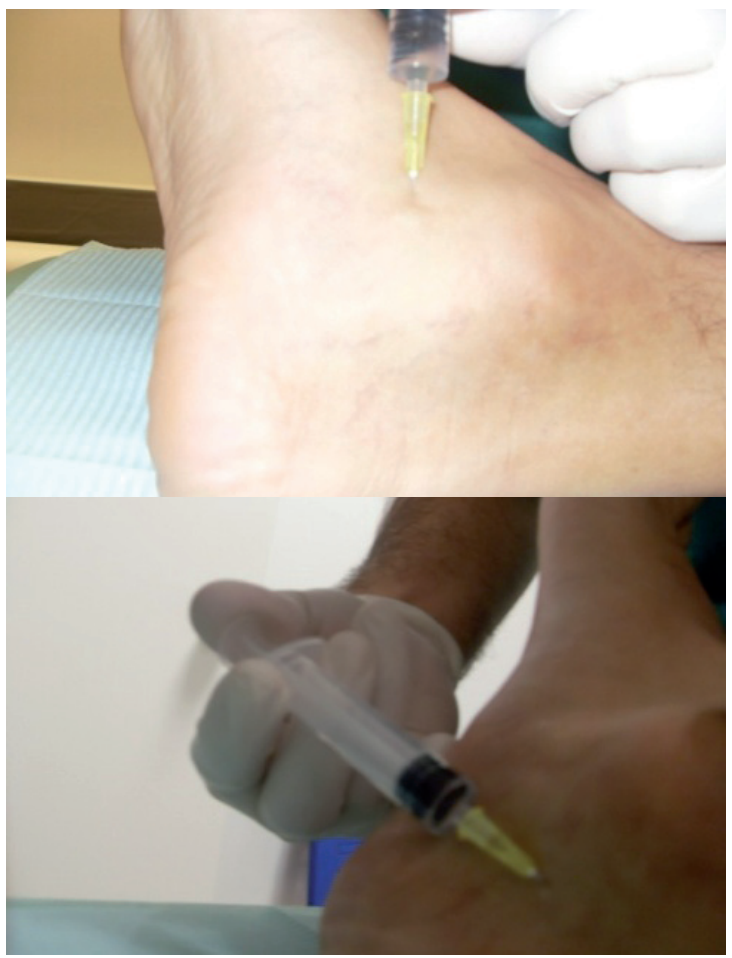

Ilustración 3. Infiltración del Tendón del Tibial Posterior a lo largo de su recorrido distal y zona de entesis.

Tabla 4. Evolución del paciente a lo largo del año de tratamiento y valoración personal.

\begin{tabular}{|lllllllll}
\hline MES & T & ES & TS & KNEE & PAIN AREAS & TP & \multicolumn{2}{ll}{ TNS VAS } \\
\hline 1 & 50 & 15 & PS & NEG & BANDA ILIOTIBIAL & DOLOR & PS & 8 \\
2 & 60 & 15 & PS & NEG & & DOLOR & NEG & 7 \\
\hline 3 & 75 & 15 & NEG & PS & & NO DOLOR & NEG & 8 \\
4 & 75 & 15 & PS & NEG & & SOBRECARGA & NEG & 6 \\
\hline 5 & 90 & 15 & NEG & NEG & & NO DOLOR & NEG & 8 \\
\hline 6 & 120 & 15 & NEG & PS & BANDA ILIOTIBIAL & SOBRECARGA & NEG & 6 \\
\hline 7 & 75 & 15 & NEG & NEG & & NO DOLOR & NEG & 8 \\
8 & 75 & 15 & NEG & NEG & & NO DOLOR & NEG & 8 \\
\hline 9 & 90 & 15 & NEG & NEG & & NO DOLOR & NEG & 8 \\
\hline 10 & 90 & 15 & NEG & PS & & NO DOLOR & NEG & 8 \\
\hline 11 & 90 & 15 & NEG & NEG & & NO DOLOR & NEG & 8 \\
\hline 12 & 120 & 15 & NEG & NEG & BANDA ILIOTIBIAL & SOBRECARGA & NEG & 8 \\
\hline
\end{tabular}


terapia.Se hacen dos controles, el primero tras esas cuatro primeras sesiones, en los que valoramos la evolución del proceso inflamatorio y la clínica del paciente. Al cabo de un año y tras la aparición de diferentes zonas de dolor referido que fueron siendo tratadas según se presentaban, el paciente estaba asintomático.

\section{CONCLUSIONES}

El tratamiento lo más precoz posible de cualquier proceso inflamatorio es un objetivo terapéutico en Medicina/Podología Deportiva. La modulación de la cascada inflamatoria asegura el desencadenamiento de todos aquellos procesos necesarios para la reparación tisular $\mathrm{y}$, por tanto, de la recuperación funcional del segmento lesionado. La inflamación, lejos de la visión clásica que aparecía como un proceso dañino y eliminable, muestra todo su potencial sanador si el proceso es llevado dentro de los márgenes fisiológicos. La Medicina Biológica nos aporta esta posibilidad al actuar en el proceso durante la fase celular y la estimulación de población linfocitaria T-reguladora, necesaria para la reparación tisular. En estudios comparativos, el medicamento biorregulador muestra una eficacia comparable a otros "antiinflamatorios" como el ibuprofeno, para la reducción del edema y del dolor, mostrando una ventaja muy amplia en tolerabilidad y seguridad de manejo. La infiltración es un método sencillo, que actúa a diferentes niveles (inmunológico, neurovascular y segmental) con escasa molestias para el paciente que, además, podemos reforzar con medicamento en soporte oral para el consumo del deportista entre las sesiones de infiltración. $\mathrm{Ni}$ en nuestra experiencia clínica de doce años manejando esta técnica, ni la bibliografía consultada han mostrado efectos secundarios, más allá del propio "pinchazo", es decir los efectos derivados de la técnica de infiltración, ni interacciones con otros medicamentos.

\section{BIBLIOGRAFÍA}

1. The EdUReP model for nonsurgical management of tendinopathy. Physical Therapy 2005;10 (85): 1093-1103.

2. Nabil J. Khoury, Georges Y. El-Khoury, Charles L. Saltzman, Eric A. Brandser. MR imaging of Posterior Tibial Tendon Dysfunction. AJR 1996; 167: 675-682.

3. Kornelia Kulig et al. Non operative management of posterior tibialis tendon dysfunction: design of a randomized clinical trial. BMC Musculoskeletal Disorders. 2006; 7:49.

4. Yasuda T, Poole AR. A fibronectin fragment induces type II collagen degradation by colagenase through an interleukin-1-mediated pathway. Arthritis Rheum 2002; 46: 138-48.

5. Leon Chaitow, Judit Walter DeLany. Aplicación clínica de técnicas neuromusculares, Tomo I. Ed. Paidotribo 2006, pag 71.

6. Alta Smit. Tratamiento de las enfermedades músculoesqueléticas. Journal of Biological Medicine 2004; 2: 53-60. 